\title{
Determining the difference in the efficacy and safety of self-expandable metallic stents as a bridge to surgery for obstructive colon cancer among patients in the CROSS 0 group and those in the CROSS 1 or 2 group: a pooled analysis of data from two Japanese prospective multicenter trials
}

\author{
Takeshi Ohki $^{1,2}$ (D) Shuntaro Yoshida ${ }^{2,3} \cdot$ Masakazu Yamamoto $^{1} \cdot$ Hiroyuki Isayama $^{2,4} \cdot$ Tomonori Yamada $^{2,5}$. \\ Takeaki Matsuzawa ${ }^{2,6} \cdot$ Shuji Saito ${ }^{2,7} \cdot$ Toshio Kuwai $^{2,8} \cdot$ Masafumi Tomita $^{2,9} \cdot$ Toshiyasu Shiratori $^{2,10}$. \\ Mamoru Shimada ${ }^{2,11} \cdot$ Tomio Hirakawa ${ }^{2,12} \cdot$ Koichi Koizumi ${ }^{2,13} \cdot$ Yoshihisa Saida ${ }^{2,14}$
}

Received: 15 October 2019 / Accepted: 16 January 2020 / Published online: 6 February 2020

(c) The Author(s) 2020

\begin{abstract}
Purpose This study compared the feasibility and safety of endoscopic placement of self-expandable metallic stents (SEMSs) as a bridge to surgery (BTS) between patients with obstructive colorectal cancer (CRC) classified as ColoRectal Obstruction Scoring System (CROSS) 0 and those with CROSS 1 or 2.

Methods We conducted a post hoc analysis of two prospective, observational, single-arm multicenter clinical trials and performed a pooled analysis of the data. In total, 336 consecutive patients with malignant colorectal obstruction underwent SEMS placement. The primary endpoint was clinical success, defined as resolution of symptoms and radiological findings within 24 h. Secondary endpoints were technical success and adverse events.

Results High clinical (98.0\% vs. 98.4\%) and technical (96.7\% vs. 97.8\%) success rates were observed in both groups (CROSS 0 vs. CROSS 1 or 2). The adverse event rate was low. The mean stricture length was lower $(3.8 \pm 1.2 \mathrm{~cm}$ vs. $4.4 \pm 1.8 \mathrm{~cm})$ and laparoscopic surgery more common (56.7\% vs 52.2\%) in the CROSS 0 group than in the CROSS 1 and 2 group.

Conclusion This study was the first to compare the degree of stricture in different CROSS groups and demonstrated comparable results with respect to the short-term efficacy and safety of SEMS placement as a BTS for obstructive CRC in CROSS 0,1 , and 2 patients.
\end{abstract}

Keywords Colorectal cancer $\cdot$ Colorectal obstruction $\cdot$ Bridge to surgery $\cdot$ Self-expandable metallic stents $\cdot$ Pooled analysis

\section{Abbreviations \\ ASA American Society of Anesthesiologists \\ BTS Bridge to surgery \\ COBRA Colonic stent for "Bridge to Surgery" pro- spective randomized controlled trial compar- ing treatment with non-stenting surgery in stage II/III obstructive colon cancer}

This work was presented at the 25th Japan Digestive Disease Week 2017, October 12-15, 2017, Fukuoka, Japan.

Members of the Japan Colonic Stent Safe Procedure Research Group are listed in "Acknowledgment".

Takeshi Ohki

ohki.takeshi@twmu.ac.jp

Extended author information available on the last page of the article
CRC Colorectal cancer

CROSS ColoRectal Obstruction Scoring System

DFS Disease-free survival

ECM Extra-colonic malignancy

ECOG Eastern Cooperative Oncology Group

ESGE European Society of Gastrointestinal Endoscopy

JCSSPRG Japan Colonic Stent Safety Procedure

Research Group

PS Performance status

RCT Randomized controlled trials

SD Standard deviations

SEMS Self-expandable metallic stents

UMIN University Hospital Medical Information Network 


\section{Introduction}

Endoscopic placement of self-expandable metal stents (SEMSs) has two major indications: to relieve the symptoms of bowel obstruction and restore the bowel function in a palliative setting and to achieve bowel decompression as a bridge to surgery (BTS). Therefore, when patients with colorectal cancer (CRC) with acute obstruction undergo radical resection, they need a diverting stoma to reduce the risk of anastomotic leakage. Recently, with the advent of SEMS placement before radical surgery, stomas have not necessarily been required [1].

Clinical guidelines from the European Society of Gastrointestinal Endoscopy (ESGE) state that SEMSs for BTS may not be safe in colonic obstruction associated with malignancy, especially on the left side of the colon [2]. This recommendation is based on the findings of various randomized controlled trials (RCTs) and cohort studies indicating that the success rate of using a SEMS as BTS was as low as $47 \%$ $(47-100 \%)$, while the perforation rate was as high as $8.7 \%$ (0-12.8\%) [3-11]. Therefore, SEMS placement for oncological indications appears to affect the long-term prognosis.

In Japan, SEMS placement has been covered by national health insurance since 2012. The Japan Colonic Stent Safety Procedure Research Group (JCSSPRG) was founded with the sole purpose of ensuring the safe use of SEMSs in Japan. Our organization began our efforts by modifying the scoring system for the assessment of the alimentation status of patients with malignant gastric outlet obstruction [12] to include clinical features of obstruction along with the oral intake status. This modified system is called the ColoRectal Obstruction Scoring System (CROSS) and uses a point score system, as shown in Table 1. CROSS 0 patients need emergency surgery or SEMS placement. CROSS 1 or 2 patients are candidates for elective surgery. CROSS 3 and 4 patients can receive food; therefore, SEMS placement is not necessary. The CROSS is widely accepted for the evaluation of the severity of obstruction in Japan. In simple terms, colonic

Table 1 The ColoRectal obstruction scoring system

\begin{tabular}{ll}
\hline Level of oral intake & Score \\
\hline Requiring continuous decompression & 0 \\
No oral intake & 1 \\
Liquid or enteral nutrient intake & 2 \\
Soft solids, low-residue, and full diet with symptoms of & 3 \\
$\quad$ stricture $^{\text {a }}$ & \\
Soft solids, low-residue, and full diet without symptoms of $^{\text {stricture }}$ & 4 \\
\hline
\end{tabular}

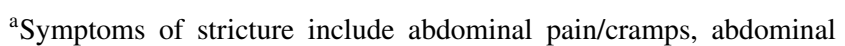
distension, nausea, vomiting, constipation, and diarrhea, which are related to gastrointestinal transit stenting for CROSS 0 patients seems to be more difficult than that for CROSS 1 or 2 patients. Thus far, however, there has been no study comparing the effectiveness and safety of SEMS placement in the CROSS 1 or 2 group and CROSS 0 group.

Our group recently launched a phase III RCT called the colonic stent for "Bridge to Surgery" prospective randomized controlled trial (COBRA). We compared this method to the treatment with non-stenting surgery in stage II/III obstructive colon cancer in order to investigate whether or not decompression by colonic stenting as a BTS for obstructive CRC is non-inferior to surgery (considered the standard treatment) [13]. This trial was registered with the University Hospital Medical Information Network (UMIN) Clinical Trial Registry (UMIN-CTR000026158), and the long-term prognosis is assessed in terms of the three-year disease-free survival (DFS), which is an index of the oncological outcome of CRC after curative resection. Of note, however, this trial only includes patients with obstructive CRC in CROSS 1 and 2 categories.

To extrapolate the results of this study to CROSS 0 patients as well, the present study aimed to clarify whether or not the effectiveness and safety of SEMS placement as a BTS in the CROSS 0 group were comparable to those in the CROSS 1 or 2 group.

\section{Methods}

\section{Study design and population}

A post hoc analysis of two prospective, observational, single-arm multicenter clinical trials involving SEMSs were carried out. One clinical trial registered with the University Hospital Medical Information Network Clinical Trial Registry (UMIN000007953) was conducted from June 2012 to October 2013 using the WallFlex colonic stent (Boston Scientific, Natick, MA, USA). The other clinical trial (UMIN000011304) was conducted from October 2013 to May 2014 using the Niti-S colonic stent (Taewoong Medical, Gimpo-si, Gyeonggi-do, South Korea).

Before these trials began, a website (https://colon-stent .com/) with information regarding the standard methods of SEMS placement based on previously published data was launched. A workshop was conducted to develop guidelines for a standard, adequate, and safe SEMS placement procedure. More than 140 physicians across Japan participated, and several of them offered their experiences to this end. This was followed by discussions among physicians at the participating facilities. The core points of these discussions were consolidated into a brief guideline that was uploaded to the website. 
Patients with acute colorectal obstruction or symptomatic strictures secondary to malignant neoplasms were enrolled at 43 participating facilities (14 academic centers and 29 community hospitals) under these two trials. Approval was obtained from the respective institutional review boards of each participating facility before the start of the trials. At the time of patient enrollment, the treatment objective (BTS or palliative) was determined based on the stage of malignant disease, coexisting illness, age, and (in some cases) patient choice.

\section{Exclusion criteria and endpoints}

Cases with previous colonic SEMS placement, enteral ischemia, suspected or impending perforation, intra-abdominal abscess, contraindications to endoscopy, and the use of a SEMS for indications other than those outlined under the trial were excluded. Patients were followed for a period of seven days.

Clinical success was the primary endpoint of this study, defined as an improvement in obstructive symptoms resulting in the resumption of food intake within $24 \mathrm{~h}$ of SEMS placement. Technical success was the secondary endpoint, defined as successful deployment of a SEMS to cover the full length of the stricture on the first attempt with no adverse events. The clinical success of BTS was defined as improvement in obstructive symptoms before surgery without any stent-related adverse events or need for endoscopic re-intervention or emergency surgery.

Adverse events included stent migration, perforation, bleeding, a fever, abdominal pain, tenesmus, and primary anastomosis with or without a diverting stoma. Complete obstruction was diagnosed when any of the following was present: inability to pass flatus, lack of water-soluble contrast passing proximal to the lesion on contrast enema, or lack of an endoscopically visible proximal lumen on colonoscopy [14-16].

\section{Type of SEMSs and the placement procedure}

Patients were treated using either an uncovered WallFlex colonic stent or a Nits-S colonic stent. WallFlex stents are available in 2 external diameters (22 and $25 \mathrm{~mm}$ ) in 3 different lengths $(6,9$, and $12 \mathrm{~cm})$. Niti-S stents are also available in 2 external diameters ( 18 and $22 \mathrm{~mm}$ ) and 4 different lengths $(6,8,10$, and $12 \mathrm{~cm})$.

The procedure for SEMS placement was as follows: a guidewire was used to establish access through the stricture, and a contrast tube was inserted into the proximal lumen. Fluoroscopy was used to measure the length of the stricture and determine the number of stents required to cross the stricture. Intraluminal or extraluminal marking using an endoscopic clip, lipoid, or radiopaque marker was performed as instructed by the endoscopist in order to locate the stricture. No dilation of the stricture was done before SEMS placement.

\section{Statistical analyses}

All analyses were performed using the JMP software program (ver. 12.2.0; SAS Institute, Chicago, IL, USA). Continuous variables are expressed as the means and standard deviations (SD), while nominal variables are described using numbers and percentages. Continuous variables were compared using the Kruskal-Wallis test, and nominal variables were compared using the Chi-squared test. A $P$ value of $<0.05$ was considered significant. Multivariate analyses were performed to study the effects of various risk factors on clinical failure.

\section{Results}

Of the 723 consecutive patients enrolled in the study, 9 were excluded due to deterioration of respiratory status $(n=1)$, usage of another type of SEMS $(n=1)$, fistula into the stomach $(n=1)$, adhesive small bowel obstruction $(n=1)$, and loose stenosis $(n=5)$ (Fig. 1$)$. Thus, the per-protocol cohort comprised the remaining 714 patients.

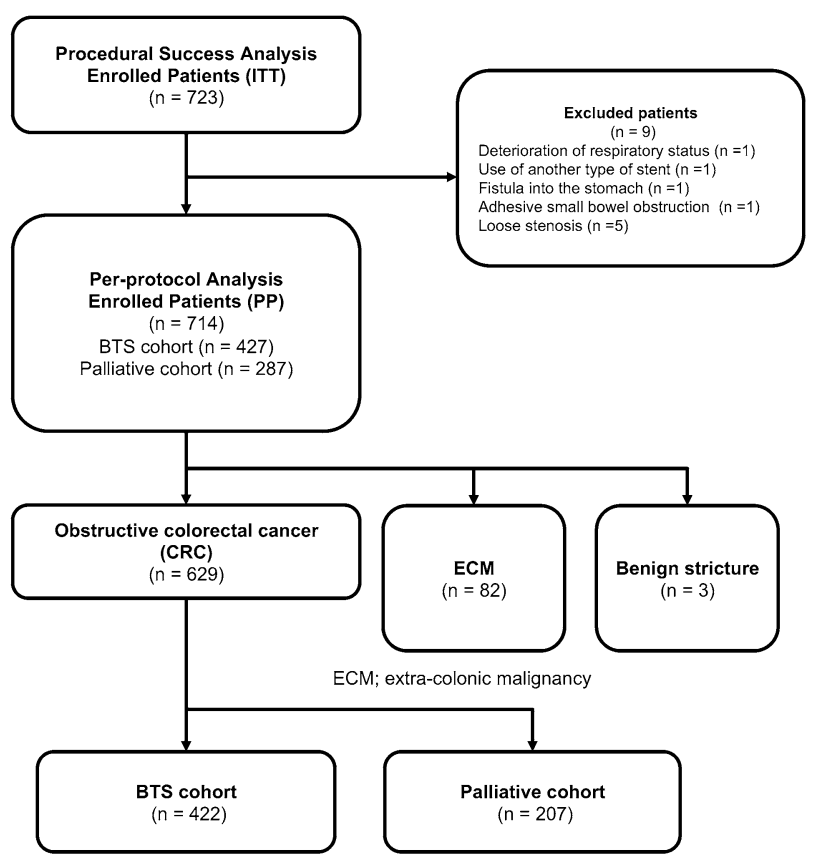

Fig. 1 Flowchart of the study showing the number of patients enrolled who had obstructive CRC. BTS bridge to surgery, $C R C$ colorectal cancer, ECM extra-colonic malignancy, ITT intention to treat, $P P$ per-protocol 
Of these patients, 629 had obstructive CRC, 82 had extracolonic malignancy (ECM), and 3 had benign stricture. Of the 629 patients with obstructive $\mathrm{CRC}$, the treatment objective was BTS in $422(62.1 \%)$ patients and palliation in the remaining 207 (32.9\%) patients.

Of the 422 BTS patients, 153 (36\%) were graded as CROSS 0, 183 (43\%) as CROSS 1 or 2, $54(13 \%)$ as CROSS 3 , and $32(8 \%)$ as CROSS 4 . Of these, CROSS 3 and 4 patients were excluded from the analysis as shown in Fig. 2. Thus, the "integrated cohort" comprised 336 patients. To evaluate adverse events after SEMS placement, all but six technically unsuccessful cases were defined as the "technical success cohort" in Fig. 2.

\section{Patient characteristics}

Table 2 shows the characteristics and details of the clinical presentation of the patients included in the study. The mean age of the patients was 69.7 years old, and male patients comprised $57.7 \%$ of the cohort $(n=194)$. Symptoms of obstruction were present in $334(99.4 \%)$ patients and included a deteriorating defecatory pattern (94.3\%), bloating $(88.1 \%)$, abdominal pain or cramps $(81.0 \%)$, and nausea or vomiting (54.2\%). Of the 336 patients, 334 (99.4\%) had primary CRC, 1 had dissemination of CRC, and 1 had local recurrence of CRC. Left-sided obstruction was more common than right-sided $(241,71.7 \%)$, and the sigmoid colon was the most common site of obstruction (106,

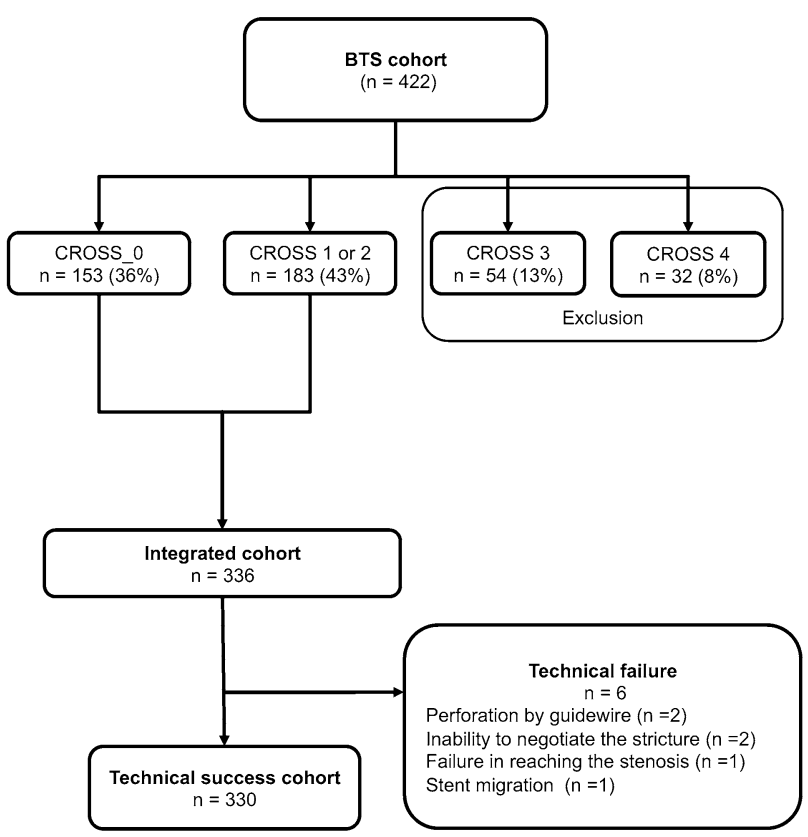

Fig. 2 Study profile showing the number of patients in each CROSS group and the derivation of the technical success cohort. CROSS ColoRectal Obstruction Scoring System
Table 2 Characteristics and details of clinical presentation of patients included in the "integrated cohort" $(n=336)$

\begin{tabular}{|c|c|}
\hline \multicolumn{2}{|l|}{ Patient characteristics } \\
\hline Age, years & $69.7 \pm 11.6$ \\
\hline Sex, male/female & $194 / 142,(57.7 / 42.3)$ \\
\hline Symptoms of obstruction present & $334(99.4)$ \\
\hline Deteriorating defecatory pattern & $317(94.3)$ \\
\hline Bloating & $296(88.1)$ \\
\hline Abdominal pain/cramps & $281(83.6)$ \\
\hline Nausea/vomiting & $182(54.2)$ \\
\hline \multicolumn{2}{|l|}{ Tumor origin } \\
\hline Primary colorectal cancer (CRC) & $334(99.4)$ \\
\hline Dissemination of CRC & $1(0.3)$ \\
\hline Local recurrence of CRC & $1(0.3)$ \\
\hline Site of obstruction, left/right & $241 / 95,(71.7 / 28.3)$ \\
\hline \multicolumn{2}{|l|}{ Tumor localization $(n=338)$} \\
\hline Rectum & $10(3.0)$ \\
\hline Rectosigmoid junction & $36(10.7)$ \\
\hline Sigmoid colon $^{\mathrm{a}}$ & $106(31.5)$ \\
\hline Sigmoid-descending colon junction & $33(9.8)$ \\
\hline Descending colon $^{\mathrm{a}}$ & $59(17.6)$ \\
\hline Splenic flexure & $14(4.2)$ \\
\hline Transverse colon & $43(12.8)$ \\
\hline Hepatic flexure & $9(2.7)$ \\
\hline Ascending colon & $17(5.1)$ \\
\hline Cecum & $11(3.3)$ \\
\hline \multicolumn{2}{|l|}{ Associated conditions } \\
\hline Localized tumor & $246(73.2)$ \\
\hline Liver metastasis & $53(15.8)$ \\
\hline Lung metastasis & $22(6.5)$ \\
\hline Peritoneal carcinomatosis & $31(9.2)$ \\
\hline Other metastases & $48(14.3)$ \\
\hline Ascites & $68(20.2)$ \\
\hline Complete obstruction $^{\mathrm{b}}$ & $303(90.2)$ \\
\hline \multicolumn{2}{|l|}{ Number of sites of obstruction } \\
\hline 1 & $335(99.7)$ \\
\hline 2 & $1(0.3)$ \\
\hline Stricture length, cm & $4.1 \pm 1.6$ \\
\hline \multicolumn{2}{|l|}{ ECOG performance status } \\
\hline 0 & $135(40.1)$ \\
\hline 1 & $153(45.5)$ \\
\hline 2 & $21(6.3)$ \\
\hline 3 & $21(6.3)$ \\
\hline 4 & $6(1.8)$ \\
\hline \multicolumn{2}{|l|}{ ASA performance status } \\
\hline 1 & $181(53.9)$ \\
\hline 2 & $133(39.5)$ \\
\hline 3 & $20(6.0)$ \\
\hline 4 & $2(0.6)$ \\
\hline 5 & $0(0)$ \\
\hline \multicolumn{2}{|l|}{ CROSS } \\
\hline 0 & $153(45.5)$ \\
\hline
\end{tabular}


Table 2 (continued)

\begin{tabular}{ll}
\hline Patient characteristics & \\
\hline 1 & $127(37.8)$ \\
2 & $56(16.7)$ \\
Treatment history & \\
Chemotherapy & $15(5)$ \\
Radiotherapy & $0(0)$ \\
\hline
\end{tabular}

Values are the mean \pm standard deviation or $\mathrm{n}(\%)$

ASA American Society of Anesthesiologists, CROSS ColoRectal Obstruction Scoring System, ECOG Eastern Cooperative Oncology Group

${ }^{a}$ One patient had two obstruction sites at the sigmoid and descending colon. The other patient had a long obstruction from the sigmoiddescending junction to the descending colon

${ }^{b}$ Complete obstruction was diagnosed when any of the following was present: inability to pass flatus, lack of water-soluble contrast passing proximal to the lesion, and lack of an endoscopically visible proximal lumen

$31.5 \%)$. Other associated conditions were localized tumor $(246,73.2 \%)$, liver metastasis $(52,15.5 \%)$, lung metastasis $(22,6.5 \%)$, peritoneal carcinomatosis $(31,9.2 \%)$, and other metastases $(48,14.3 \%)$. Ascites was present in $68(20.2 \%)$ patients. Complete obstruction was present in $303(90.2 \%)$ patients. All but 1 had a single obstruction $(442,99.7 \%)$. The mean stricture length was $4.1 \mathrm{~cm}$. The classification of patients on the basis of their Eastern Cooperative Oncology Group (ECOG) performance status (PS) was as follows: 135 (40.1\%) patients were classified as PS 0, $153(45.5 \%)$ as PS 1, $21(6.3 \%)$ as PS 2, 21(6.3\%) as PS 3, and $6(1.8 \%)$ as PS 4. Regarding the American Society of Anesthesiologists (ASA) physical status classification of patients, 181 (53.9\%) patients were classified as ASA 1, $133(39.6 \%)$ as ASA 2, 20 $(6.0 \%)$ as ASA 3, and $2(0.5 \%)$ as ASA 4. Fifteen patients $(5 \%)$ underwent chemotherapy before SEMS placement.

\section{Interventions before SEMS placement and procedural details}

Digestive tract decompression using a tube was performed in 98 patients $(29.2 \%)$ at the time of the diagnosis (Table 3 ), while stricture balloon dilation was performed in $6(1.8 \%)$ patients. As preparation for SEMS placement, 114 patients (33.9\%) were administered cleansing enema. Intraluminal and/or extraluminal stricture marking was done in 241 $(71.7 \%)$ patients. The technical failure rate (where an SEMS could not be placed in a stricture) was $1.9 \%(n=6)$. Of the remaining 330 patients in whom stents were successfully deployed, $322(97.6 \%)$ had a single stricture with 1 stent, $7(2.1 \%)$ had a single stricture with 2 stents, and $1(0.3 \%)$ had a double stricture with 2 stents. In total, 338 stents were deployed, of which WallFlex colonic stents constituted
Table 3 Interventions before SEMS placement and procedural details of patients included in the "integrated cohort" $(n=336)$

\begin{tabular}{|c|c|}
\hline \multicolumn{2}{|l|}{ Interventions before SEMS placement } \\
\hline $\begin{array}{l}\text { Digestive tract decompression before SEMS place- } \\
\text { ment }\end{array}$ & $98(29.2)$ \\
\hline Naso-gastric tube & $22(6.5)$ \\
\hline Naso-intestinal tube & $38(11.3)$ \\
\hline Trans-anal tube & $41(12.2)$ \\
\hline Stricture balloon dilation & $6(1.8)$ \\
\hline \multicolumn{2}{|l|}{ Preparation for SEMS placement } \\
\hline Cleansing enema & $114(33.9)$ \\
\hline Oral bowel cleansing & $16(4.8)$ \\
\hline Stricture marking done & $241(71.7)$ \\
\hline Intraluminal & $221(65.8)$ \\
\hline Extraluminal & $25(7.4)$ \\
\hline \multicolumn{2}{|l|}{ Details of SEMS procedure } \\
\hline \multicolumn{2}{|l|}{ Strictures and stents placed } \\
\hline Stricture with no stent (technical failure) & $6(1.9)$ \\
\hline Single stricture with 1 stent & $322 / 330(97.6)$ \\
\hline Single stricture with 2 stents & $7 / 330(2.1)$ \\
\hline Double stricture with 2 stents & $1 / 330(0.3)$ \\
\hline \multicolumn{2}{|l|}{ Stent type (total number of stents deployed, $338^{\mathrm{a}}$ ) } \\
\hline WallFlex colonic stent & $248(73.4)$ \\
\hline Niti-S colonic stent & $90(26.6)$ \\
\hline $6 \mathrm{~cm}$ long & $179(53.0)$ \\
\hline $8 \mathrm{~cm}$ long & $36(10.7)$ \\
\hline $9 \mathrm{~cm}$ long & $87(25.7)$ \\
\hline $10 \mathrm{~cm}$ long & $22(6.5)$ \\
\hline $12 \mathrm{~cm}$ long & $14(4.2)$ \\
\hline $18 \mathrm{~mm}$ diameter & $11(3.3)$ \\
\hline $22 \mathrm{~mm}$ diameter & $309(91.4)$ \\
\hline $25 \mathrm{~mm}$ diameter & $18(5.3)$ \\
\hline Procedure time in the technical success cohort, min & $37.9 \pm 20.4$ \\
\hline Technical success rate & $330(98.2)$ \\
\hline Clinical success rate & $327(97.3)$ \\
\hline Clinical success rate of BTS & $305(90.8)$ \\
\hline
\end{tabular}

Values are the mean \pm standard deviation or $n(\%)$

$S E M S$ self-expandable metallic stent, BTS bridge to surgery

${ }^{a}$ Denominator for calculating the stent percentages

$73.4 \%$ ( $n=248)$. The $22 \mathrm{~mm}$ stents were the most commonly used stents $(309,91.4 \%)$. The mean procedure time for stent placement was $37.9 \mathrm{~min}$.

The technical success rate and clinical success rate were $98.2 \%$ and $97.3 \%$, respectively (Table 3 ). However, following successful stent placement, adverse events occurred in 22 patients, bringing the clinical success rate of BTS to $90.8 \%$. Some of the adverse events observed following SEMS placement included perforation in three patients, stent migration in two patients, and stent occlusion in one patient. Perforation related to guidewire manipulation was observed 
in one patient in the CROSS 1 and 2 groups. There were two stent-related perforations. Perforation caused by appendicitis related to stent placement was observed in one patient in the CROSS 1 and 2 groups. Perforation at the tumor site was observed in one patient in the CROSS 0 group. No additional stent placement for migration was needed. Although stent re-obstruction caused by stool occurred in one patient in the CROSS 0 group, obstruction was treated with endoscopy.

\section{The comparison of CROSS 0 and CROSS 1 or 2 (Tables 4, 5, and 6)}

There was a statistically significant difference in the severity of stenosis (complete obstruction vs. incomplete obstruction) between the two groups, with more patients from the CROSS 0 group having complete obstruction (146 (95.4\%) vs. 157 (85.8\%), $P=0.003$ ) (Table 4). Although high rates of complete occlusion were shown (95.4\% in the CROSS 0 group and $85.8 \%$ in the CROSS 1
Table 5 A comparison of the adverse events in colonic stenting between CROSS 0 and CROSS 1 or 2 patients in the "technical success cohort" $(n=330)$

\begin{tabular}{llll}
\hline Adverse events $^{\mathrm{a}}$ & $\begin{array}{l}\text { CROSS 0 } \\
(n=150)\end{array}$ & $\begin{array}{l}\text { CROSS 1 or 2 } \\
(n=180)\end{array}$ & \\
\hline $\begin{array}{l}\text { Early adverse events } \\
(<7 \text { days })\end{array}$ & $9(6.0)$ & $16(9.0)$ & 0.32 \\
Stent migration & 0 & $2(1.1)$ & 0.20 \\
Perforation & $1(0.7)$ & $2(1.1)$ & 0.67 \\
Stent occlusion & 0 & $1(0.7)$ & 0.27 \\
Bleeding & $1(0.7)$ & $3(1.7)$ & 0.41 \\
Fever & $1(0.7)$ & $1(0.6)$ & 0.90 \\
Abdominal pain & $3(2.0)$ & $4(2.2)$ & 0.89 \\
Tenesmus & $2(1.3)$ & $2(1.1)$ & 0.85 \\
Others & $5(3.3)$ & $5(2.8)$ & 0.77 \\
\hline
\end{tabular}

CROSS ColoRectal Obstruction Scoring System

${ }^{\text {a }}$ Patients without technical success were excluded
Table 4 A comparison of CROSS 0 and CROSS 1 or 2 patients included in the "integrated cohort" $(n=336)$

\begin{tabular}{|c|c|c|c|}
\hline & CROSS $0(n=153)$ & CROSS 1 or $2(n=183)$ & $P$ value \\
\hline Age, years & $70.1 \pm 11.9$ & $69.4 \pm 11.2$ & 0.31 \\
\hline Sex, male/female & $94 / 59(61.4 / 38.6)$ & $100 / 83(54.6 / 45.4)$ & 0.21 \\
\hline Symptoms of obstruction present & $153(100)$ & $181(98.9)$ & 0.19 \\
\hline Left-sided obstruction & $105(68.6)$ & $136(74.3)$ & 0.25 \\
\hline Complete obstruction $^{\mathrm{a}}$ & $146(95.4)$ & $157(85.8)$ & 0.003 \\
\hline Localized tumor & $114(74.5)$ & $132(72.1)$ & 0.62 \\
\hline Stricture length, $\mathrm{cm}$ & $3.8 \pm 1.2$ & $4.4 \pm 1.8$ & 0.002 \\
\hline $\begin{array}{l}\text { Digestive tract decompression before } \\
\text { SEMS placement }\end{array}$ & $71(46.1)$ & $27(14.8)$ & $<0.0001$ \\
\hline ECOG performance status & & & 0.16 \\
\hline 0 & $67(43.8)$ & $68(37.2)$ & \\
\hline 1 & $59(38.6)$ & $94(51.4)$ & \\
\hline 2 & $11(7.2)$ & $10(5.5)$ & \\
\hline 3 & $13(8.5)$ & $8(4.4)$ & \\
\hline 4 & $3(2.0)$ & $3(1.6)$ & \\
\hline ASA performance status & & & 0.18 \\
\hline 1 & $75(49.0)$ & $106(57.9)$ & \\
\hline 2 & $70(45.8)$ & $63(34.4)$ & \\
\hline 3 & $7(4.6)$ & $13(7.1)$ & \\
\hline 4 & $1(0.7)$ & $1(0.6)$ & \\
\hline 5 & 0 & 0 & \\
\hline Technical success rate & $150(98.0)$ & $180(98.4)$ & 0.82 \\
\hline Clinical success rate of BTS & $139(90.9)$ & $166(90.7)$ & 0.97 \\
\hline Procedural time, min & $39.1 \pm 21.3$ & $37.2 \pm 19.7$ & 0.41 \\
\hline
\end{tabular}

Values are the mean \pm standard deviation or $\mathrm{n}(\%)$

ASA American Society of Anesthesiologists, CROSS ColoRectal Obstruction Scoring System, ECOG Eastern Cooperative Oncology Group, SEMS self-expandable metallic stent, BTS bridge to surgery

${ }^{\mathrm{a}}$ Complete obstruction was diagnosed when any of the following was present: inability to pass flatus, lack of water-soluble contrast passing proximal to the lesion, and lack of an endoscopically visible proximal lumen 
Table 6 A comparison of the surgical details, mortality, and morbidity between CROSS 0 and CROSS 1 or 2 patients in the "Technical success cohort" $(n=330)$

\begin{tabular}{llll}
\hline & CROSS_0 (n=150) & $\begin{array}{l}\text { CROSS 1 or 2 } \\
(n=180)\end{array}$ \\
\hline Type of surgery & & $78(43.3)$ & 0.049 \\
Open surgery & $50(33.3)$ & $94(52.2)$ & \\
Laparoscopic surgery & $85(56.7)$ & $8(4.4)$ & \\
Conversion & $15(10.0)$ & $163(90.6)$ & 0.49 \\
Primary anastomosis & $139(92.7)$ & $4(2.2)$ & 0.79 \\
Primary anastomosis with diverting stoma & $4(2.7)$ & $21.6 \pm 17.0$ & 0.51 \\
Time to surgery after stenting, d & $20.4 \pm 16.2$ & $15.8 \pm 12.9$ & 0.80 \\
Time to discharge after surgery, d & $16.1 \pm 12.0$ & 0 & 0.46 \\
Mortality & $1(0.3)$ & & \\
Morbidity & & 0 & 0.50 \\
Bleeding & 0 & $2(1.1)$ & 0.79 \\
Anastomotic leakage requiring for surgery & 0 & $7(3.9)$ & 0.13 \\
Anastomotic leakage treated by conservative therapy & $7(4.7)$ & $6(3.3)$ & 0.18 \\
Intra-abdominal abscess & $1(0.6)$ & $1(0.6)$ & 0.21 \\
Wound infection & $4(2.6)$ & 0 & 0.21 \\
Postoperative intestinal obstruction & $2(1.3)$ & 0 & \\
Respiratory comorbidity & $2(1.3)$ & & \\
\hline
\end{tabular}

CROSS ColoRectal Obstruction Scoring System

${ }^{\text {a}}$ Patients without technical success were excluded or 2 group), there was a statistically significant difference between the 2 cohorts. The mean stricture length was found to be significantly lower in the CROSS 0 group than in the CROSS 1 or 2 group $(3.8 \pm 1.2 \mathrm{~cm}$ vs. $4.4 \pm 1.8 \mathrm{~cm}$, $P=0.002)$. Digestive tract decompression before SEMS placement was performed in significantly more patients classified as CROSS 0 than in those classified as CROSS 1 or 2 (71 [46.1\%] vs. 27 [14.8\%], $P<0.0001)$. There was no significant difference in the technical and clinical success rates between the two groups.

The two groups were comparable with respect to the age, sex, tumor location, ECOG and ASA status, technical/clinical success rates, and procedural times, (Tables 4 and 5). Regarding the types of surgery, laparoscopic surgery was performed more frequently in the CROSS 0 group than in the CROSS 1 or 2 group (56.7\% vs. $52.2 \%, P=0.049)$. In addition, the primary anastomosis rates with and without diverting stoma, time to surgery, and time to discharge after surgery in both groups were comparable (Table 6). The mortality and morbidity due to surgery after stenting were also similar (Table 6).

\section{Factors associated with clinical failure}

A multivariate analysis was performed to study the effects of various risk factors on clinical failure (Table 7); it showed that complete obstruction was an independent predictive factor of clinical success.
Table 7 Results of the multivariate analysis of predictive factors for clinical failure with colonic stenting for symptomatic CRC

\begin{tabular}{llll}
\hline Risk factor & Odds ratio & $95 \%$ CI & $P$ value \\
\hline $\begin{array}{l}\text { CROSS grading (CROSS 0 } \\
\text { vs. CROSS 1 or 2) }\end{array}$ & 2.19 & $0.40-11.97$ & 0.37 \\
$\begin{array}{l}\text { Digestive tract decom- } \\
\text { pression before SEMS } \\
\text { placement }\end{array}$ & 1.83 & $0.35-9.29$ & 0.47 \\
$\begin{array}{l}\text { Complete obstruction } \\
\text { Stricture length (per cm) }\end{array}$ & 0.09 & $0.02-0.39$ & 0.001 \\
\hline
\end{tabular}

CRC colorectal cancer, $C I$ confidence interval, CROSS ColoRectal Obstruction Scoring System, SEMS self-expandable metallic stent

\section{Discussion}

This study shows that SEMS placement as a BTS for obstructive CRC has good efficacy and high safety. The JCSSPRG, established in 2012 with the sole purpose of ensuring the safe use of SEMS in Japan, conducted two large multicenter, prospective studies in relation to SEMSs, the first of its kind in Japan. In 1 study, a total of 518 patients with malignant colorectal obstruction were treated with the WallFlex colonic stent, while in the other, 205 patients were treated with the Niti-S colonic stent.

Matsuzawa et al. reported the short-term outcomes in terms of the technical success rate $(99.5 \%)$ and clinical success rate $(97.9 \%)$ of SEMS placement using a WallFlex 
colonic stent with strict inclusion criteria and stricture marking [16]. Saito et al. found that placement of the WallFlex colonic stent as a BTS for malignant colorectal obstruction was a feasible approach [17]. Similarly, our group also noted good results when using a Niti-S colonic stent $[18,19]$. Tomita et al. concluded that SEMS placement for malignant colorectal obstruction as a BTS was safe and effective with respect to peri-procedural outcomes using a pooled analysis of two prospective cohort studies [20].

In the present study, we used the same pooled data as Tomita et al. [21], but instead of placing all patients in one big group, we performed a post hoc analysis to compare the safety and efficacy of SEMS placement as a BTS for obstructive CRC in CROSS 0 with those in CROSS 1 and 2. As mentioned above, patients classified as CROSS 0 were clinically in a more critical state than those classified as CROSS 1 and 2. Contrary to expectations, both groups showed a high clinical success rate (98.0\% vs. $98.4 \%)$ as well as a high technical success rate $(96.7 \%$ vs. $97.8 \%)$. Thus, SEMS placement as a BTS for obstructive CRC had good efficacy and high safety in both CROSS 0 and CROSS 1 and 2 patients. Interestingly, complete obstruction was found to be associated with a favorable outcome in terms of the clinical success of colonic stenting.

One particularly important point concerning the present study was our speculation of the cause of the difference in the outcomes of colonic stent procedures between the two groups (complete cases vs. incomplete cases). Unfortunately, we did not evaluate how the bowel was prepared during the procedure in this study. Regarding the visibility of the obstruction site during colonoscopy, it is higher if patients have a complete obstruction as the feces flow from the proximal side to the distal side at the obstruction site is almost entirely cut off in complete obstruction cases. On the other hand, in incomplete obstruction cases, the flow of feces will impair the visibility.

Our ongoing phase III randomized control trial, called the COBRA trial, seeks to verify whether or not decompression with colonic stenting for obstructive CRC is non-inferior to surgery in terms of the disease-free survival (DFS) of stage II/III CRC at three years after primary tumor resection. However, only obstructive CRC patients in CROSS 1 and 2 categories are included in that trial. CROSS 0 patients were excluded because emergency surgery is usually needed in those patients. Given their poor physical status and lifethreatening condition, performing primary anastomosis in such patients is considered dangerous. Acute resection for obstructive left-sided colon cancer in an emergency setting results in a high colostomy rate (61.4\%) and high risk of conversion to a more invasive approach, such as open surgery (90.8\%) [22]. Consequently, the high colostomy (often permanent type) rate decreases the quality of life of patients
[23]. We previously reported a high primary anastomosis rate $(92 \%)$ in a prospective clinical trial on colonic stenting as BTS for obstructive CRC [20]. Taken together, these present and previous findings suggest that it is difficult to obtain informed consent from CROSS 0 patients in this regard.

In addition, stent insertion for obstructive CRC may result in an increase in CK20 mRNA [21], cell-free DNA, circulating tumor DNA [24], and viable circulating tumor cells (v-CTCs) [25]. To accurately evaluate the effect of tumor manipulation by the radial force generated when a SEMS expands, the influence of additional surgery, such as colostomy, should be avoided as much as possible. However, excluding CROSS 0 patients (who are expected to have severe strictures) may reduce the number of cases with adverse events caused by colonic stenting, resulting in an overestimation of the effectiveness and safety of stenting compared to surgery. Therefore, the current study compared the effectiveness and safety of SEMS placement in the CROSS 1 or 2 group and CROSS 0 group.

A longer stricture length and fewer laparoscopic surgeries may be expected in the CROSS 0 group than in the CROSS 1 or 2 group; however, we found that the mean stricture length was $0.6 \mathrm{~cm}$ shorter in the CROSS 0 group, and the percentage of laparoscopic surgeries was $4.5 \%$ higher. We suspect that this was because of the high number of CRC cases classified as macroscopic type with wall stricture sign (also known as stricture-type CRC) among these patients. Stricture-type CRC looks like a "bow tie" owing to the marked fold convergence of the intestinal tract, causing more than $30 \%$ wall shrinkage [26]. Boku et al. showed that the stricture was caused by a high amount of intestinal fibrosis, which might lead to cancer invasion [27]. Despite being smaller than other types, stricture-type CRC can be easily spotted and resected by laparoscopic surgery because of its unique shape. These findings suggest that patients in the CROSS 0 group probably had this type of tumor. Other studies have also found that stricture-type CRC had a considerably higher recurrence rate and shorter recurrence period in the colorectum than other types.

The conversion rate of laparoscopic surgery was $10 \%$ in the CROSS 0 group and $4.4 \%$ in the CROSS 1 or 2 group. The results of the JCOG 0404 study [28] showed that laparoscopic surgery had a low conversion rate (5.4\%) in Japan; however, the CROSS 0 group tended to show a higher rate than the CROSS 1 or 2 groups. The conversion rate of laparoscopic surgery was higher than the average in Japan because obstructive CRC cases were considered. Laparoscopic surgery is more difficult in cases with obstruction than in those without due to inflammation, strong adhesion, and handling of edematous intestine. The CROSS 0 group may require more careful selection for laparoscopic surgery. Park et al. [29] reported that the conversion rate of laparoscopic surgery was $35.3 \%$ in the 
emergency surgery group and $4.3 \%$ in the SEMS group; the CROSS 1 or 2 group showed similar but lower results for both conversion rates.

Several limitations associated with the present study warrant mention. First, it was a a non-comparative study with no control group. Furthermore, the long-term outcomes were not studied, and the study was confined to Japan.

In conclusion, two large multicenter, prospective studies demonstrated the short-term efficacy and safety of SEMS placement as a BTS for patients with obstructive CRC classified as CROSS 0, 1, and 2. Furthermore, the effectiveness and safety of SEMS placement as a BTS in the CROSS 0 group were shown to be comparable to those in the CROSS 1 or 2 group.

Acknowledgements This study was supported by the Japan Gastroenterological Endoscopy Society.

The Japan Colonic Stent Safe Procedure Research Group includes the following members in addition to the authors: Tatsuya Osuga, Aijinkai Takatsuki General Hospital, Takatsuki, Japan; Hiroo Matsushita, Akita Red Cross Hospital, Akita, Japan; Mitsuru Goto, Asahikawa Kosei Hospital, Asahikawa, Japan; Shungo Endo, Fukushima Medical University Aizu Medical Center, Aizu-Wakamatsu, Japan; Yohei Kurose, Fukuyama City Hospital, Fukuyama, Japan; Shigeru Yamagishi, Fujisawa City Hospital, Fujisawa, Japan; Katsuya Ota, Higashiosaka City General Hospital, Higashiosaka, Japan; Masanori Yoshimitsu, Hiroshima City Asa Hospital, Hiroshima, Japan; Satoshi Ikeda, Hiroshima Prefectural Hospital, Hiroshima, Japan; Rintaro Moroi, Iwate Prefectural Isawa Hospital, Oshu, Japan; Routa Noaki, Kawaguchi Municipal Medical Center, Kawaguchi, Japan; Hirofumi Kawamoto, Kawasaki Medical School, Okayama, Japan; Hirotoshi Hasegawa, Keio University School of Medicine, Tokyo, Japan; Atsushi Yamauchi, Kitano Hospital, Osaka, Japan; Fuminori Teraishi, Kochi Health Sciences Center, Kochi, Japan; Kohei Takayasu, Kyorin University Hospital, Mitaka, Japan; Takahiro Horimatsu, Kyoto University Hospital, Kyoto, Japan; Yoshinori Kushiyama, Matsue Red Cross Hospital, Matsue, Japan; Hiroaki Naota, Matsusaka Chuo General Hospital, Matsusaka, Japan; Takuya Yamaguchi, Mimihara General Hospital, Sakai, Japan; Shigenori Masaki, Miyanomori Memorial Hospital, Sapporo, Japan; Taku Sakamoto, National Cancer Center Hospital, Tokyo, Japan; Chizu Yokoi, National Center for Global Health and Medicine, Tokyo, Japan; Masafumi Inomata, Oita University Faculty of Medicine, Oita, Japan; Nobuya Obana, Osaki Citizen Hospital, Osaki, Japan; Masayoshi Horimoto, Osaka Saiseikai Senri Hospital, Suita, Japan; Shinei Kudo, Showa University Northern Yokohama Hospital, Yokohama, Japan; Yasushi Nakamura, Takano Hospital, Kumamoto, Japan; Mitsunori Ushigome, Toho University Omori Medical Center, Tokyo, Japan; Takeshi Ohki, Tokyo Women's Medical University, Tokyo, Japan; Hiroyuki Kato, Tokyo Women's Medical University Medical Center East, Tokyo, Japan; Shiro Hayashi, Toyonaka Municipal Hospital, Toyonaka, Japan; Kensuke Kubota, Yokohama City University Hospital, Yokohama, Japan; Kunihiko Amano, Saitama Medical Center, Saitama Medical University, Moroyama, Japan; Masanori Yoshino, Nippon Medical School Musashi Kosugi Hospital, Kawasaki, Japan; Takuji Kawamura, Japanese Red Cross Kyoto Daini Hospital, Kyoto, Japan; Yorinobu Sumida, Kushu Medical Center, Fukuoka, Japan; Noriko Watanabe, Mie Chuo Medical Center, Tsu, Japan; Hideto Egashira, Shonan kamakura General Hospital, Kamakura, Japan; Takahisa Kayahara, Kurashiki Central Hospital, Kurashiki, Japan; Hideki Kanazawa, Sagamihara National Hospital, Sagamihara, Japan; Michiaki Watanabe, Atsugi City Hospital, Atsugi, Japan; and Kensuke Kubota, Yokohama City University Hospital, Yokohama, Japan.

\section{Compliance with ethical standards}

Conflict of interest Dr. Yoshida reports personal fees from Century Medical Inc., personal fees from Boston Scientific Japan, personal fees from ZEON, outside the submitted work; Dr. Isayama reports personal fees and research funding from Century Medical Inc., personal fees from Boston Scientific Corp, personal fees from Japan Lifeline Co., Ltd., during the conduct of the study; Dr. Matsuzawa reports personal fees from Century Medical Inc., during the conduct of the study; Dr. Saito reports personal fees from Century Medical Inc., personal fees from Boston Scientific Japan, personal fees from COOK Japan, personal fees from Japan Lifeline Co., Ltd., personal fees from Kawasumi Laboratories, Inc., during the conduct of the study; Dr. Kuwai reports personal fees from Olympus Medical Systems, personal fees and research funding from Century Medical Inc., personal fees from Boston Scientific Japan, during the conduct of the study; Dr. Saida reports research funding from Century Medical Inc., research from Boston Scientific Japan, research from Olympus Medical Systems, personal fees from Japan Lifeline Co., Ltd. during the conduct of the study; Dr. Ohki, Dr. Yamamoto, Dr. Yamada, Dr. Tomita, Dr. Shiratori, Dr. Shimada, and Dr. Hirakawa, and Dr. Koizumi have nothing to disclose.

Open Access This article is licensed under a Creative Commons Attribution 4.0 International License, which permits use, sharing, adaptation, distribution and reproduction in any medium or format, as long as you give appropriate credit to the original author(s) and the source, provide a link to the Creative Commons licence, and indicate if changes were made. The images or other third party material in this article are included in the article's Creative Commons licence, unless indicated otherwise in a credit line to the material. If material is not included in the article's Creative Commons licence and your intended use is not permitted by statutory regulation or exceeds the permitted use, you will need to obtain permission directly from the copyright holder. To view a copy of this licence, visit http://creativecommons.org/licenses/by/4.0/.

\section{References}

1. Cennamo V, Luigiano C, Coccolini F, Fabbri C, Bassi M, De Caro $\mathrm{G}$, et al. Meta-analysis of randomized trials comparing endoscopic stenting and surgical decompression for colorectal cancer obstruction. Int J Colorectal Dis. 2013;28:855-63.

2. van Hooft JE, van Halsema EE, Vanbiervliet G, Beets-Tan RG, DeWitt JM, Donnellan F, et al. Self-expandable metal stents for obstructing colonic and extracolonic cancer: European Society of Gastrointestinal Endoscopy (ESGE) Clinical Guideline. Endoscopy. 2014;46:990-1053.

3. Pirlet IA, Slim K, Kwiatkowski F, Michot F, Millat BL. Emergency preoperative stenting versus surgery for acute left-sided malignant colonic obstruction: a multicenter randomized controlled trial. Surg Endosc. 2011;25:1814-21.

4. Hooft JE, Bemelman WA, Oldenburg B, Marinelli AW, Lutke Holzik MF, Grubben MJ, et al. Colonic stenting versus emergency surgery for acute left-sided malignant colonic obstruction: a multicentre randomised trial. Lancet Oncol. 2011;12:344-52.

5. Ghazal AH, El-Shazly WG, Bessa SS, El-Riwini MT, Hussein AM. Colonic endolumenal stenting devices and elective surgery versus emergency subtotal/total colectomy in the management of malignant obstructed left colon carcinoma. J Gastrointest Surg. 2013;17:1123-9.

6. Tung KL, Cheung HY, Ng LW, Chung CC, Li MK. Endo-laparoscopic approach versus conventional open surgery in the treatment 
of obstructing left-sided colon cancer: long-term follow-up of a randomized trial. Asian J Endosc Surg. 2013;6:78-81.

7. Ho KS, Quah HM, Lim JF, Tang CL, Eu KW. Endoscopic stenting and elective surgery versus emergency surgery for left-sided malignant colonic obstruction: a prospective randomized trial. Int J Colorectal Dis. 2012;27:355-62.

8. Alcantara M, Serra-Aracil X, Falco J, Mora L, Bombardo J, Navarro S. Prospective, controlled, randomized study of intraoperative colonic lavage versus stent placement in obstructive left-sided colonic cancer. World J Surg. 2011;35:1904-10.

9. Sabbagh C, Browet F, Diouf M, Cosse C, Brehant O, Bartoli E, et al. Is stenting as "a bridge to surgery" an oncologically safe strategy for the management of acute, left-sided, malignant, colonic obstruction? A comparative study with a propensity score analysis. Ann Surg. 2013;258:107-15.

10. Sloothaak DAM, Van Den Berg MW, Dijkgraaf MGW, Fockens P, Tanis PJ, Van Hooft JE, et al. Oncological outcome of malignant colonic obstruction in the Dutch Stent-In 2 trial. Br J Surg. 2014;101:1751-7.

11. Gorissen KJ, Tuynman JB, Fryer E, Wang L, Uberoi R, Jones OM, et al. Local recurrence after stenting for obstructing left-sided colonic cancer. Br J Surg. 2013;100:1805-9.

12. Adler DG, Baron TH. Endoscopic palliation of malignant gastric outlet obstruction using self-expanding metal stents: experience in 36 patients. Am J Gastroenterol. 2002;97:72-8.

13. University Hospital Medical Information Network Clinical Trial Registry. Tokyo. UMIN Clinical Trials Registry; [about 10 screens]. https://upload.umin.ac.jp/cgi-open-bin/ctr/ctr_view. cgi?recptno=R000029703. Accessed 23 Jun 2019.

14. Small AJ, Coelho-Prabhu N, Baron TH. Endoscopic placement of self-expandable metal stents for malignant colonic obstruction: long-term outcomes and complication factors. Gastrointest Endosc. 2010;71:560-72.

15. Yoshida S, Watabe H, Isayama H, Kogure H, Nakai Y, Yamamoto N, et al. Feasibility of a new self-expandable metallic stent for patients with malignant colorectal obstruction. Dig Endosc. 2013;25:160-6.

16. Matsuzawa $\mathrm{T}$, Ishida $\mathrm{H}$, Yoshida $\mathrm{S}$, Isayama $\mathrm{H}$, Kuwai $\mathrm{T}$, Maetani I, et al. A Japanese prospective multicenter study of self-expandable metal stent placement for malignant colorectal obstruction: short-term safety and efficacy within 7 days of stent procedure in 513 cases. Gastrointest Endosc. 2015;82:697-707.

17. Saito S, Yoshida S, Isayama H, Matsuzawa T, Kuwai T, Maetani I, et al. A prospective multicenter study on self-expandable metallic stents as a bridge to surgery for malignant colorectal obstruction in Japan: efficacy and safety in 312 patients. Surg Endosc. 2016;30:3976-86.

18. Narita A, Yoshida S, Isayama H, Yamada T, Maetani I, Sumida $\mathrm{Y}$, et al. Prospective multicenter study of a flexible selfexpandable metallic stents for malignant colorectal obstruction in Japan: short-term safety and efficacy in 199 patients. 23rd UEG Week
2015. 2015; Barcelona, Spain: October 26. United Eur Gastroenterol J. 2015;3:A267.

19. Matsuzawa T, Ishida H, Yamada T, Tomita M, Yoshida S, Isayama $\mathrm{H}$, et al. Prospective multicenter study of selfexpandable metallic stent placement as a bridge to surgery for malignant colorectal obstruction in Japan: feasibility in 112 cases. 23rd UEG Week 2015. 2015; Barcelona, Spain: October 26. United Eur Gastroenterol J. 2015;3:A585.

20. Tomita M, Saito S, Makimoto S, Yoshida S, Isayama H, Yamada $\mathrm{T}$, et al. Self-expandable metallic stenting as a bridge to surgery for malignant colorectal obstruction: pooled analysis of 426 patients from two prospective multicenter series. Surg Endosc. 2019;33:499-509.

21. Tanis PJ, Pereira NRP, van Hooft JE, Consten ECJ, Bemelman WA. Resection of obstructive left-sided colon cancer at a national level: a prospective analysis of short-term outcomes in 1816 patients. Dig Surg. 2015;32:317-24.

22. Deans GT, Krukowski ZH, Irwin ST. Malignant obstruction of the left colon. Br J Surg. 1994;81:1270-6.

23. Maruthachalam K, Lash GE, Shenton BK, Horgan AF. Tumour cell dissemination following endoscopic stent insertion. Br J Surg. 2007;94:1151-4.

24. Takahashi G, Yamada T, Iwai T, Takeda K, Koizumi M, Shinji S, et al. Oncological assessment of stent placement for obstructive colorectal cancer from circulating cell-free DNA and circulating tumor DNA dynamics. Ann Surg Oncol. 2018;25:737-44.

25. Yamashita S, Tanemura M, Sawada G, Moon J, Shimizu Y, Yamaguchi T, et al. Impact of endoscopic stent insertion on detection of viable circulating tumor cells from obstructive colorectal cancer. Oncol Lett. 2018;15:400-6.

26. Boku N, Yoshida S, Ohtsu A, Fujii T, Koba I, Oda Y, et al. Expression of integrin $\alpha 3$ in gastric and colorectal cancers: its relation to wall contraction and mode of invasion. Jpn J Cancer Res. 1955;86:934-40.

27. Miyamoto S, Boku N, Fujii T, Ohtsu A, Matsumoto S, Tajiri H, et al. Macroscopic typing with wall stricture sign may reflect tumor behaviors of advanced colorectal cancers. J Gastroenterol. 2001;36:158-65.

28. Yamamoto S, Inomata M, Katayama H, Mizusawa J, Etoh T, Konishi F, et al. Short-term surgical outcomes from a randomized controlled trial to evaluate laparoscopic and open D3 dissection for stage II/III colon cancer: Japan Clinical Oncology Group Study JCOG 0404. Ann Surg. 2014;260:23-30.

29. Park SJ, Lee KY, Kwon SH, Lee SH. Stenting as a bridge to surgery for obstructive colon cancer: does it have surgical merit or oncologic demerit? Ann Surg Oncol. 2016;23:842-8.

Publisher's Note Springer Nature remains neutral with regard to jurisdictional claims in published maps and institutional affiliations.

\section{Affiliations}

\section{Takeshi Ohki ${ }^{1,2}$ (1) - Shuntaro Yoshida ${ }^{2,3} \cdot$ Masakazu Yamamoto $^{1} \cdot$ Hiroyuki Isayama ${ }^{2,4} \cdot$ Tomonori Yamada $^{2,5}$. Takeaki Matsuzawa ${ }^{2,6} \cdot$ Shuji Saito ${ }^{2,7} \cdot$ Toshio Kuwai $^{2,8} \cdot$ Masafumi Tomita $^{2,9} \cdot$ Toshiyasu Shiratori $^{2,10}$. Mamoru Shimada ${ }^{2,11} \cdot$ Tomio Hirakawa $^{2,12} \cdot$ Koichi Koizumi $^{2,13} \cdot$ Yoshihisa Saida ${ }^{2,14}$}

1 Department of Surgery, Institute of Gastroenterology, Tokyo Women's Medical University, 8-1 Kawada-cho, Shinjuku-ku, Tokyo 162-8666, Japan
2 Japan Colonic Stent Safe Procedure Research Group, Tokyo, Japan 
3 Department of Endoscopy and Endoscopic Surgery, Graduate School of Medicine, The University of Tokyo, Tokyo, Japan

4 Department of Gastroenterology, Graduate School of Medicine, Juntendo University, Tokyo, Japan

5 Department of Gastroenterology, Japanese Red Cross Nagoya Daini Hospital, Nagoya, Japan

6 Department of Surgery, IMS Miyoshi General Hospital, Miyoshi, Japan

7 Division of Surgery, Gastrointestinal Center, Yokohama Shin-Midori General Hospital, Yokohama, Japan

8 Department of Gastroenterology, National Hospital Organization Kure Medical Center and Chugoku Cancer Center, Kure, Japan

9 Department of Surgery, Kishiwada Tokushukai Hospital, Osaka, Japan
10 Department of Gastroenterology, Kameda Medical Center, Kamogawa, Japan

11 Department of Surgery, Toyonaka Midorigaoka Hospital, Osaka, Japan

12 Department of Gastroenterology, Toyonaka Midorigaoka Hospital, Osaka, Japan

13 Department of Gastroenterology, Tokyo Metropolitan Cancer and Infectious Disease Center Komagome Hospital, Tokyo, Japan

14 Department of Surgery, Toho University Ohashi Medical Center, Tokyo, Japan 